\title{
Understanding the space-time properties of the deconfined phase in ultrarelativistic heavy-ion collisions.
}

\author{
Adam Kisiel*i \\ Department of Physics, Ohio State University, \\ 1040 Physics Research Building, \\ 191 West Woodruff Ave., Columbus, OH 43210, USA \\ E-mail: kisiel@mps.ohio-state.edu
}

\begin{abstract}
The collisions of ultrarelativistic heavy-ions at RHIC (BNL) show evidence that a deconfined phase of quarks and gluons is produced there. The system is believed to rapidly evlolve, and go through a transition to the ordinary hadronic matter. The femtoscopy technique is able, through the analysis of two-particle correlations, to measure the space-time extent of the system at the point of hadron emission, and probe its dynamics. Hydrodynamic models aim to describe the behaviour of the system and provide predictions for both the system size and its dependence on the evolution dynamics. I present recent femtoscopic results from STAR experiment at RHIC and confront them with hydrodynamic predictions. I also show extrapolations to the LHC regime and present predictions for the ALICE experiment.
\end{abstract}

8th Conference Quark Confinement and the Hadron Spectrum September 1-6 2008

Mainz, Germany

\footnotetext{
* Speaker.

${ }^{\dagger}$ This work was supported by the U.S. NSF grant no. PHY-0653432.
} 


\section{Introduction}

The goal of Heavy-Ion Collisions at ultra-relativistic energies, performed at the Relativistic Heavy-Ion Collider (RHIC) in Brookhaven National Laboratory and, in the near future, at the Large Hadron Collider at CERN is to create matter in a deconfined phase, called Quark-Gluon Plasma. Its discovery has been announced by the RHIC experiments [1, 2, 3, 4]. It was found to behave as a perfect fluid. One of the major pieces of evidence to support this claim was the fact the the momentum properties of the bulk system (momentum spectra, radial and elliptic flow) appear to be properly described by hydrodynamics $[5,6]$.

Femtoscopy is a technique which uses correlations between the momenta of two particles to probe the space-time behavior of the system which emits them [7]. RHIC has produced a large and detailed set of femtoscopic data. Initially the hydrodynamic models mentioned above had a difficulty to describe these data. However recently a consensus is emerging that these problems were a consequence of using several assumptions, which had to be modified in order to reproduce femtoscopic data.

Below we show some of the femtoscopic data from RHIC which are then interpreted with the help of the Lhyquid and Therminator model $[8,9,10]$, which incorporates hydrodynamic expansion as well as statistical hadronization and strong resonance decay. We also show predictions for the LHC energies made by extrapolating the model parameters.

\section{Therminator+Lhyquid model}

Hydrodynamics has been used to model heavy-ion reactions, because it is expected that the system created there will behave collectively. Since we focus on the femtoscopic observables we require a model which provides freeze-out coordinated of particles. Approximately $2 / 3$ of pions created in HIC are produced via decay of resonances. This has important implications for nonidentical particle correlations, because it introduces additional emission asymmetries in time. In this work we used the Lhyquid hydrodynamic model [9, 10], with Gaussian initial conditions and realistic equation of state with no first-order phase transition. The parameters were the same as in [10] for central collisions, which we consider here. The Lhyquid model was subsequently coupled to Therminator [8] program which simulated resonance propagation and decay. No hadronic rescattering is employed. We use the two-particle method described in detail in [11] to calculate correlation functions. This ensures that they are calculated in a manner as closely resembling the experimental one as possible.

\section{Collectivity signals in femtoscopy}

The femtoscopic correlation function $C$ is defined as a ratio of probabilities to observe a pair of particles in coincidence (in the same event) to the one to observe such pair independently (in two different events). This ratio is analyzed as a function of pair relative momentum $\vec{q}$. If we assume the absence of other two-particle correlations, then $C$ can be related to the source emission function $S$ : 


$$
C(\vec{q})=\int S_{1,2}(\mathbf{r}, \vec{q}) \Psi_{1,2}(\vec{q}, \mathbf{r}) d^{4} r
$$

The indexes 1,2 correspond to particle types analyzed (since particle types are known, their masses are fixed, hence the relative momentum $\vec{q}$ has only three independent components). Emission function $S$ can be interpreted as a probability to emit a pair of particles of a given type with relative momentum $\vec{q}$ at a relative distance $\mathbf{r}$. $\Psi$ is the relative wave-function of the given twoparticle system and is the combination of the Coulomb interaction (if both 1 and 2 are charged), strong interaction (for hadrons) and proper (anti-)symmetrization of the wave function (if 1 and 2 are identical).

Femtoscopic information is obtained by assuming some functional, parametric form of $S$, then performing the integral (3.1) (analytically if possible, numerically otherwise) and comparing it to the measured correlation function. Parameters of $S$ that yield the best fit to the experimental $C$ are the result of the analysis.

Most common femtoscopic correlation function for HIC are the ones for identical charged pions. They are especially useful because of large pion multiplicities observed, which enable analysis as a function of many variables. In this work we will concentrate on the dependence on pair transverse momentum $k_{T}=\frac{1}{2}\left(p_{T, 1}+p_{T, 2}\right)$. The most common parametrization of $S_{\pi \pi}$ is a $3 \mathrm{~d}$ sphere with Gaussian density profile, with the three sigmas $R$ along three axis as parameters. The directions are defined as follows: long along the beam axis, out along the pair transverse momentum and side perpendicular to the other two.

Another useful femtoscopic correlation involves non-identical particles [13]. In this work we will concentrate on pion-kaon correlations due to the high multiplicities of these particles, but similar considerations can be performed for pion-proton and kaon-proton pairs. Pair multiplicities are significantly smaller than for identical pions, which means that no $k_{T}$ dependence study is possible. The interaction is Coulomb dominated and its directional sensitivity is small, so only the determination of the overall source size $R_{i n v}$ is possible. However due to the fact that particles are not identical one more crucial observable is available. Whereas for identical particles the first moment of $S$ is by construction equal to 0 and only the second moments - the "sizes" $R$ are accessible, for non-identical particles the first moments - "shifts" are also measurable. Therefore the femtoscopic information from pion-kaon correlations are: overall system size $R$ and shift(s) between average emission points of pions and kaons.


Figure 1: Emission points of primordial pions from the Therminator model (central RHIC collisions), as a function of particle transverse momentum (from left to right: $0.15-0.25 \mathrm{GeV} / \mathrm{c}, 0.25-0.35 \mathrm{GeV} / \mathrm{c}, 0.35-0.45$ $\mathrm{GeV} / \mathrm{c}, 0.45-0.6 \mathrm{GeV} / \mathrm{c}$ ) 
Hydrodynamic models of HIC predict that the matter created in such collisions undergoes an explosive expansion from the initial "pancake", created by the two colliding nuclei. The flows in the longitudinal direction (along the beam axis) are strong because the initial gradients are largest. But the transverse expansion is also significant. We concentrate on the transverse direction. The collective flow of matter produces a specific ordering of $p_{T}$ spectra of particles with different masses, which is observed in HICs. It also produces a distinct dependence of the transverse femtoscopic radii $R_{\text {out }}$ and $R_{\text {side }}$ on $k_{T}$ - both are expected to fall as $k_{T}$ increases. It is illustrated in Fig. 1, where emission points of pions with different $p_{T}$ are plotted. The monotonic decrease of RMS'es of these distributions both in "side" and "out" directions are observed - producing the $k_{T}$ dependence mentioned above. However there is an alternative scenario, not involving hydrodynamics, which could produce a similar $k_{T}$ dependence: the higher $p_{T}$ particles are emitted from a hot an small core, while lower $p_{T}$ particles - from larger and cooler corona. In this picture the source does not have a "collective" velocity and emits particles from each of its points in all directions. Where this picture differs qualitatively from the "hydrodynamic" one is in the average emission points. The collective flow in hydrodynamics not only makes the "faster" particles be emitted from apparently smaller regions in space, but also predicts that average emission points of particles with larger $p_{T}$ will be shifted more towards the edge of the system. Here is where the crucial new piece of information from non-identical particle femtoscopy comes into play. The pion and kaon which have small relative momentum in their rest frame have the same velocity, and therefore very different $p_{T}$ 's in the lab frame. Therefore their average emission points are expected to be different, which is exactly what is probed by the femtoscopic analysis. Detailed considerations show that for rapidity symmetric systems expected at RHIC and the LHC, the average shift in the long direction should vanish, while side vanishes due to symmetry. Only the out shift is expected to be non-zero and is expected to show that pions are on the average emitted closer to the center of the system than kaons. This is illustrated in Fig. 2 where emission points of pions and kaons with the same velocity (falling within the $p_{T}$ acceptance of STAR TPC) are plotted. This time one should concentrate on the means of the distributions - they are different for pions and kaons in the "out" direction (and 0 in "side") and clearly show heavier particles to be emitted closer to the edge of the system.
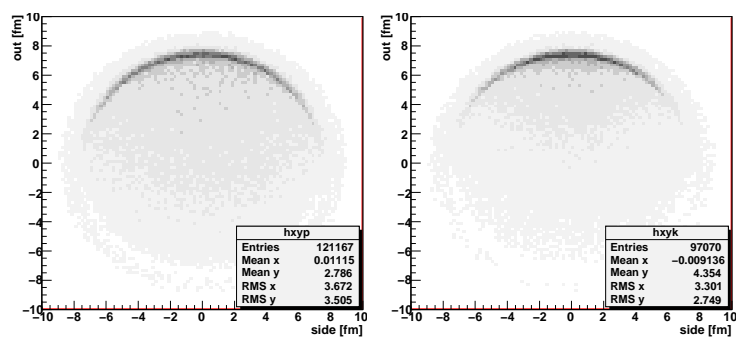

Figure 2: Emission points of primordial pions and kaons from the Therminator model (central RHIC collisions), for velocity range 0.55 to 0.8 .

\section{Comparison with data}

The femtoscopic analysis has been an integral part of the research program at RHIC. In this work we compare the predictions from the Lhyquid+Therminator models to the data. The femto- 
scopic results are a prediction in a the sense that all the parameters of the model have been fixed to reproduce other observables (integrated multiplicities, spectra and v2 of pions, kaons, protons).
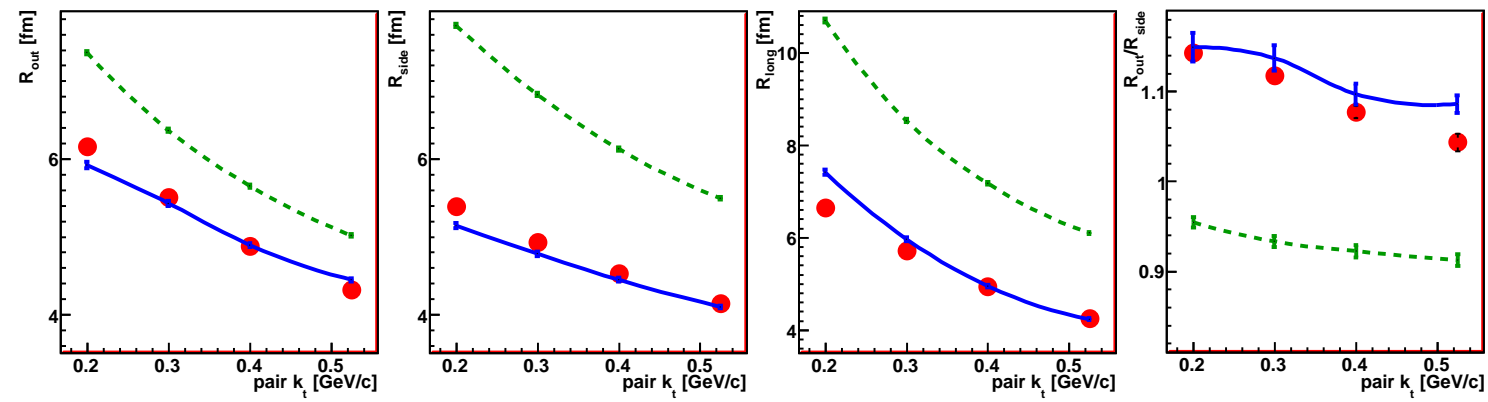

Figure 3: STAR femtoscopic radii[14] for pions (points) compared to model predictions (solid line). Model predictions for LHC energies is also shown (dashed line).

In Fig. 3 we show the traditional HBT radii vs. pair transverse momentum $k_{T}$ for central collisions. The results from the data [14] are well reproduced, only the longitudinal radius $R_{\text {long }}$ is slightly overestimated, due to the approximate treatment of longitudinal evolution of the system. Most importantly the $R_{\text {out }} / R_{\text {side }}$ ratio, which was always difficult to model correctly in hydrodynamics, is well reproduced. The agreement is a result of at least three factors: a Gaussian initial condition leading to fast development of radial flow in the initial phase, the realistic equation of state with no first-order phase transition, and a complete treatment of hadronic resonances. All these features modify the $R_{\text {out }} / R_{\text {side }}$ ratio in the same direction, making it compatible with data.

Based on the success of the HBT radii reproduction for RHIC, a prediction for LHC is also shown in Fig. 3. One can see an overall increase of the system size as well as steeper slope of out and side radii, indicating stronger radial flows. $R_{\text {out }} / R_{\text {side }}$ ratio is predicted to decrease below unity, a consequence of a change in freeze-out configuration from "outside-in" to "inside-out" [12].
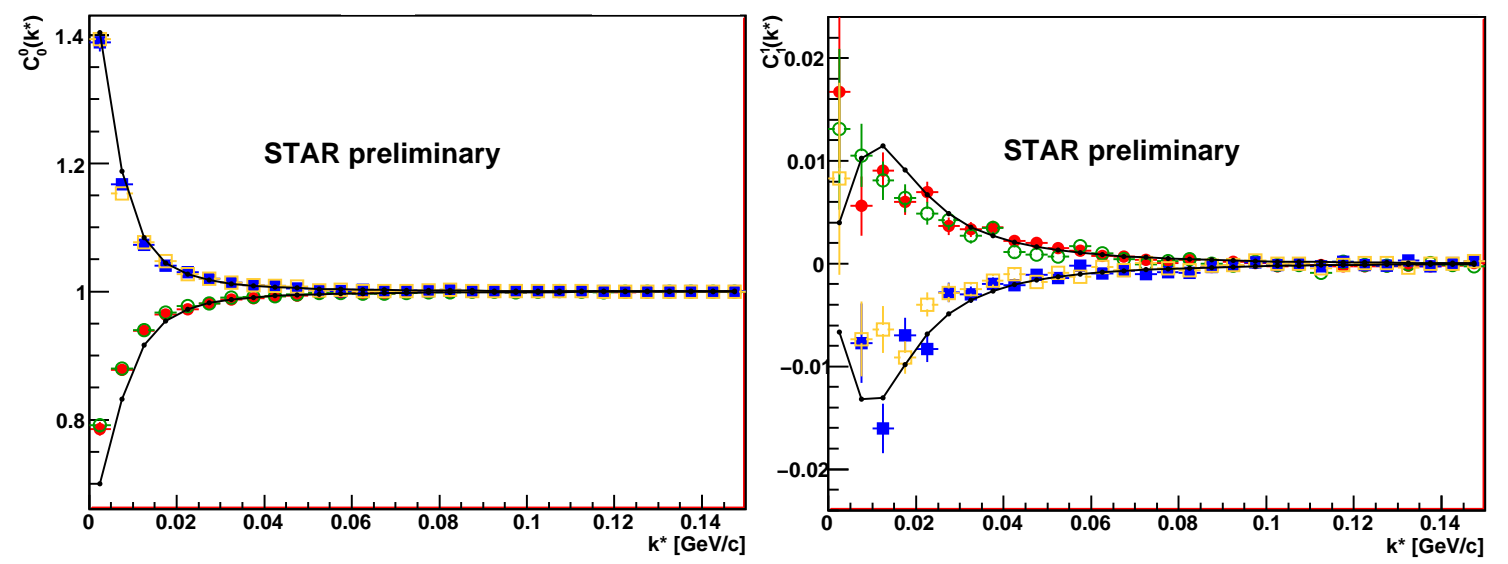

Figure 4: STAR pion-kaon (filled circles $-\pi^{+} K^{+}$, open circles $\pi^{-} K^{-}$, filled squares $-\pi^{+} K^{-}$, open squares $\pi^{-} K^{+}$) correlation functions (left panel) and asymmetry signal (right panel) compared to the Lhyquid+Therminator prediction (solid lines).

Fig. 4 shows the preliminary results for pion-kaon correlations from STAR (points) compared to the calculation from Therminator+Lhyquid (lines). The data are corrected for pair purity, while 
realistic momentum resolution smearing is applied to the model curve, so the two should be directly comparable. One can see a rather good quantitative agreements of the correlation function $\left(C_{0}^{0}\right)$ as well as the asymmetry signal $\left(C_{1}^{1}\right)$. Curves for same-sign and unlike-sign combinations agree well with each other, and follow expected trends (Coulomb repulsion/attraction respectively). The asymmetry signal in data is significant and shows that kaons are emitted closer to the edge of the system and/or earlier. Therminator+Lhyquid model is able to reproduce it both qualitatively and quantitatively. It shows that hydrodynamics is fully consistent with the non-identical particle femtoscopy, while other models which do not include collective behavior of matter are strongly challenged by this result.

\section{Summary}

We have shown selected femtoscopic signals observed in the STAR experiment. These were interpreted with the help of Therminator+Lhyquid model as evidence for collective behavior of matter resulting in strong radial flow. The $k_{T}$ dependence of the system size as well as relative shifts between average emission points of particles with different masses are reproduced by the model. Model parameters have been extrapolated to the LHC energies and predictions for femtoscopic observables have been shown.

\section{References}

[1] J. Adams et al. [STAR Collaboration], Nucl. Phys. A 757, 102 (2005) [arXiv:nucl-ex/0501009].

[2] B. B. Back et al., Nucl. Phys. A 757, 28 (2005) [arXiv:nucl-ex/0410022].

[3] I. Arsene et al. [BRAHMS Collaboration], Nucl. Phys. A 757, 1 (2005) [arXiv:nucl-ex/0410020].

[4] K. Adcox et al. [PHENIX Collaboration], Nucl. Phys. A 757, 184 (2005) [arXiv:nucl-ex/0410003].

[5] U. W. Heinz, J. Phys. G 31 (2005) S717 [arXiv:nucl-th/0412094].

[6] T. Hirano and K. Tsuda, Phys. Rev. C 66 (2002) 054905 [arXiv:nucl-th/0205043].

[7] M. A. Lisa, S. Pratt, R. Soltz and U. Wiedemann, Ann. Rev. Nucl. Part. Sci. 55 (2005) 357 [arXiv:nucl-ex/0505014].

[8] A. Kisiel, T. Taluc, W. Broniowski and W. Florkowski, Comput. Phys. Commun. 174, 669 (2006) [arXiv:nucl-th/0504047].

[9] M. Chojnacki, W. Florkowski, W. Broniowski and A. Kisiel, Phys. Rev. C 78, 014905 (2008) [arXiv:0712.0947 [nucl-th]].

[10] W. Broniowski, M. Chojnacki, W. Florkowski and A. Kisiel, Phys. Rev. Lett. 101 (2008) 022301 [arXiv:0801.4361 [nucl-th]].

[11] A. Kisiel, W. Florkowski and W. Broniowski, Phys. Rev. C 73, 064902 (2006) [arXiv:nucl-th/0602039].

[12] A. Kisiel, W. Broniowski, M. Chojnacki and W. Florkowski, arXiv:0808.3363 [nucl-th].

[13] J. Adams et al. [STAR Collaboration], Phys. Rev. Lett. 91 (2003) 262302 [arXiv:nucl-ex/0307025].

[14] J. Adams et al. [STAR Collaboration], Phys. Rev. C 71 (2005) 044906 [arXiv:nucl-ex/0411036]. 